\title{
Vermittlung qualitativer Forschungsmethoden im digitalen Raum
}

\author{
(Didaktische) Einblicke in eine bisher wenig thematisierte \\ Praxis der Hochschullehre
}

André Epp

\section{Zusammenfassung des Beitrags}

Im Rahmen der akademischen Bildung wird der Vermittlung qualitativer Forschungsmethoden disziplinübergreifend eine gesteigerte Relevanz beigemessen. Ungeachtet dessen liegen jedoch bisher nur wenige didaktische Entwürfe zu ihrer Vermittlung via Online-Medien vor. In dem Beitrag wird daher anhand einer Einführungsvorlesung zu qualitativen Forschungsmethoden ${ }^{1}$ und einer Forschungswerkstatt zur biografischen Fallrekonstruktion veranschaulicht, wie und mit welchen (didaktischen) Rahmungen qualitative Methodenlehre im digital vermittelten Raum gelingen kann.

Schlüsselbegriffe: Digitale Lebre $\bullet$ Qualitative Forschungsmethoden $\bullet$ Flipped-Classroom $\bullet$ Forschungswerkstatt

\section{Relevanz qualitativer Methodenausbildung im Hochschulstudium}

Die Bedeutung, die der qualitativen Methodenausbildung im Hochschulstudium zukommt, nimmt disziplin- und fachübergreifend zu. Zum einen sollen Studierende in die Lage versetzt werden, entsprechende Studien zu verstehen, kritisch zu reflektieren sowie eigene Forschungsdesigns und -vorhaben zu konzipieren und umzusetzen. Zum anderen wird mit der qualitativen Methodenausbildung ebenso die Hoffnung verbunden, dass Ausbilden von Schlüsselqualifikationen, wie ganzheitliches und analytisches Denken, von Empathie, Kommunikationsfähigkeit, Flexibilität, Verantwortungsübernahme, Ambiguitäts-, Irritations-, Frustrations- und Unsicherheitstoleranz, zu unterstützen und zu befördern sowie zur Entwicklung einer reflexiven Haltung und dem Anregen von Perspektivenwechseln beizutragen (vgl. Kunz et al. 2020; Schreier/Breuer 2020). Entsprechend kommt dem Vermitteln und Erlernen qualitativer Forschung im Rahmen akademischer Bildung pädagogischer Fachkräfte gegenwärtig eine beson-

\footnotetext{
${ }^{1}$ In der Einführungsvorlesung werden sowohl qualitative als auch quantitative Methoden vermittelt. Kurz: Die Vorlesung teilt sich in zwei Teile. In dem Beitrag wird jedoch nur auf den ersten - den qualitativen Teil - eingegangen. Daher wird nachfolgend von der Einführungsvorlesung zu qualitativen Methoden gesprochen.
} 
dere Relevanz zu, da sich davon versprochen wird das Entwickeln pädagogischer Professionalität und Reflexivität zu befördern (vgl. Schrader et al. 2020, S. 200). Daher werden zunehmend kasuistisch orientierte Lehrveranstaltungen und Formate des forschenden Lernens in die erziehungswissenschaftliche Hochschullehre implementiert, wie insbesondere in der Lehrer*innenbildung ersichtlich wird (vgl. bspw. Beutel et al. 2020; Spies/Knapp 2020). Über die Arbeit mit qualitativen Forschungsmethoden, so die Hoffnung, werden grundlegende Fähigkeiten für das zukünftige Handeln als Lehrkraft bzw. Fachkraft aufgebaut, wie bspw. der Zugang zum (Fremd-)Verstehen von Klient*innen und Schüler*innen und/oder zur Reflexion des pädagogischen Handelns und beruflicher Anforderungen befördert (vgl. Fabel-Lamla/Pietsch 2012; Schrader et al. 2020, S. 200ff.).

Vor dem Hintergrund aktueller gesellschaftlicher Entwicklungen im Zeitalter des Anthropozäns kann qualitativen Forschungsmethoden ebenso eine besondere Bedeutung zugespielt werden, da für die transformative Bewältigung von (Um-)Brüchen und tiefgreifender gesellschaftlicher Veränderungen die zuvor erwähnten Schlüsselqualifikationen als elementar bzw. als notwendiger denn je angesehen werden müssen. Daher werden gegenwärtig zunehmend Überlegungen angestellt, inwiefern Aspekte dieser Forschungsweise vermehrt in nicht sozialwissenschaftlichen Curricula, wie bspw. von künstlerisch und technisch ausgerichteten Fächern, aufzunehmen sind (vgl. Kanter/Mey 2021, S. 48).

\section{Vermittlung qualitativer Forschungsmethoden in der (Präsenz-)Lehre - Zu Rele- $\operatorname{vanz}(\mathrm{en})$ direkter Interaktionen}

Im deutschsprachigen Raum ist die qualitative Methodenlehre insgesamt bisher nur wenig empirisch erforscht (vgl. Pfaff/Tervooren 2020, S. 157). Zudem liegen weiterhin nur wenige didaktische Entwürfe vor (vgl. Breuer/Schreier 2007; Mey 2008), sodass die Diskussionen im Hinblick auf die Lehr- und Lernbarkeit qualitativer Forschung und das Einsozialisieren in eine qualitative Haltung ${ }^{2}$ insgesamt als eher verhalten zu beschreiben sind (vgl. Pfaff/Tervooren 2020, S. 157; Ruppel 2020, S. 218). Jedoch lassen sich aktuell zunehmend wichtige Auseinandersetzungen beobachten (vgl. Jütte/Walber/Lobe 2016; Kunz et al. 2020; Pfaff/Tervooren 2020), die neben grundsätzlichen Rahmungen den Blick ebenso auf spezifische Lehr- und Lernformate, wie Seminare und Vorlesungen (vgl. Pfaff/Tervooren 2020), aber auch Forschungswerkstätten (vgl. Ruppel 2020) und Tutor*innenqualifikationen (vgl. Panenka/Epp/Kunina-Habenicht 2021) werfen, „so dass qualitative Forschungsmethoden und ihre Methodologien zunehmend einen eigenständigen Platz in der Hochschuldidaktik erhalten“ (Pfaff/Tervooren 2020, S. 157).

Für die Vermittlung qualitativer Forschungsmethoden werden direkte Interaktionen und Kommunikationen als zentral angesehen (vgl. Dausien 2007, S. 22f.) - Pfaff und Tervooren

\footnotetext{
${ }^{2}$ Im Gegensatz zur rein technischen Anwendung der Methoden, wird unter dem Einsozialisieren in eine qualitative Haltung ein grundlegendes Verständnis dieser Forschungsweise verstanden, die insbesondere in Forschungswerkstätten erfolgt und ausreichender zeitlicher Kapazitäten bedarf (vgl. Kanter/Mey 2021, S. 30).
} 
(2020, S. 158) sprechen auch von einem „Angewiesen sein auf die Performanz des gemeinsamen Tuns“ -, sodass entsprechende Lehrveranstaltungen „an Hochschulen bislang in erster Linie auf klassischen präsenzorientierten [...] Lehr-Lern-Zusammenhängen“ (ebd., S. 158) basieren - wie es für die Mehrzahl an Lehrveranstaltungen im Hochschulkontext zutrifft. Aufgrund der Auswirkungen der Corona-Pandemie können seit dem Frühjahr 2020 Präsenzformate jedoch nicht mehr wie gewöhnlich realisiert werden, sodass Lehrveranstaltungen in den digitalen Raum verlegt werden mussten. Diese Auswirkungen betreffen fast alle Lehrenden qualitativer Forschungsmethoden an Hochschulen, sodass sich für sie u.a. die folgenden Fragen stellen: Wie kann qualitative Methodenlehre im digital vermittelten Raum realisiert werden? Welche Inhalte, Kenntnisse und Fähigkeiten können relativ unproblematisch weitergegeben werden und welche nicht bzw. nur bedingt? Welche Werkzeuge und Räume werden als Ersatz für die fehlende Präsenz benötigt und welche öffnenden und schließenden Momente gehen damit einher?

Da aufgrund der Dominanz präsenzorientierter Lehr-Lern-Formate bisher nur wenige didaktische Entwürfe und Diskussionen vorliegen, wie qualitative Forschungsmethoden in einer digitalisierten Umgebung gelernt und gelehrt werden können (vgl. ebd., S. 157), und dem Sachverhalt, dass die Hochschullehre seit nunmehr drei Semestern notgedrungen im digital vermittelten Raum erfolgt, zielen die nachfolgenden Ausführungen einerseits darauf ab, die formulierten Fragen (qualitativ Methodenlehrender) aufzugreifen und andererseits erste Einblicke in eine entsprechende qualitative Methodenlehre und ihre didaktische Ausgestaltung in bisher ungewohnten Settings zu geben. Zudem werden Möglichkeiten und Chancen, wie auch Herausforderungen aufgezeigt. Da sich die qualitative Methodenausbildung an Hochschulen in verschiedenen sozialräumlichen Formaten vollzieht (vgl. ebd., S. 158), wurde sich bewusst für zwei sehr unterschiedliche Lehr-Lernformate, die in den digital vermittelten Raum verlegt wurden, entschieden, die in Abschnitt 3 veranschaulicht werden: Einer Einführungsvorlesung zu qualitativen Forschungsmethoden und einer Forschungswerkstatt zur biografischen Fallrekonstruktion.

\section{Qualitative Methodenlehre im digital vermittelten Raum am Beispiel einer Vorle- sung und Forschungswerkstatt}

Die beiden fokussierten Veranstaltungsformate werden zunächst im allgemeinen Kanon der Hochschullehre verortet. Im Anschluss daran werden Einblicke in die konkrete didaktische Ausgestaltung dieser im digital vermittelten Raum an der Pädagogischen Hochschule Karlsruhe gegeben (3.1 u. 3.2).

Bei der Vorlesung handelt es sich um das älteste und das am häufigsten anzutreffende Lehrveranstaltungsformat an Hochschulen, das auch aufgrund pragmatischer Gründe angeboten wird: angesichts großer Studierendenzahlen ist es notwendig, Lehrveranstaltungen für hohe Teilnehmer*innenzahlen auszubringen. Dem Format der Vorlesung kommt fach- und disziplinübergreifend eine zentrale Stellung zu und wird jedes Semester von einer Vielzahl Studierender besucht, da dort grundlegende und zentrale Inhalte dargeboten werden (vgl. Kerres/ 
Preußler 2013), wie auch an der Vorlesung zur Einführung in qualitative Forschungsmethoden an der Pädagogischen Hochschule Karlsruhe sichtbar wird: Sie wird jedes Semester von ca. 400 Studierenden besucht, weist eine hohe Fluktuation auf und muss von allen Lehramtsstudierenden obligatorisch belegt und mit einer Klausur erfolgreich abgeschlossen werden.

Dem Format der Forschungswerkstatt wird zwar im Hinblick auf das Einsozialisieren in eine qualitative Haltung eine zentrale Relevanz zugespielt (vgl. Pfaff/Tervooren 2020), jedoch kommt ihr im Rahmen der Hochschullehre bisher nur eine marginale Bedeutung zu. Sie ist an der Pädagogischen Hochschule Karlsruhe fakultativ und nicht curricular angebunden - entsprechend muss auch keine Prüfungsleistung erbracht werden. Es stehen lediglich acht Plätze zur Verfügung und es wird in einer relativ festen Gruppe über mehrere Semester hinweg zusammengearbeitet. Im Gegensatz zur Vorlesung bekommt der Hochschullehrende für die Forschungswerkstatt keine SWS angerechnet.

\subsection{Flipped-Classroom-Vorlesung im digital vermittelten Raum}

Die Vorlesung zur Einführung in qualitative Forschungsmethoden ist im Studienverlaufsplan im dritten Fachsemester in einem Pflichtmodul der Lehramtsstudiengänge an der Pädagogischen Hochschule Karlsruhe verortet und zielt darauf ab, Studierenden einen Überblick über die Grundlagen qualitativer Forschung mit ihren theoretischen, methodischen und forschungspraktischen Rahmungen zu geben.

Im digital vermittelten Raum folgt die Vorlesung dem didaktischen Modell des Flipped-Classrooms (auch als Inverted Classroom oder umgedrehter Unterricht bezeichnet) - also einer Variante des Blended-Learnings (vgl. Owston 2013). Allerdings ist Flipped-Classroom nicht als ein klar definiertes didaktisches Konzept zu charakterisieren, sondern weist verschiedene Varianten, Formen und Strömungen auf (vgl. Abeysekera/Dawson 2014; Sein-Echaluce/Fidalgo-Blanco/García-Peñalvo 2019), in dessen Rahmen ebenso auf unterschiedliche Methoden und Online-Materialien zurückgegriffen wird (vgl. Loviscach 2020, S. 90). Eine Verbreitung hat das didaktische Modell Flipped-Classroom insbesondere im Rahmen der zunehmenden Dissemination und Implementierung digitaler Techniken, wie der Etablierung von Lernmanagementsystemen an Schulen und Hochschulen, erfahren (vgl. Schäfer 2012). Ziel ist es, dass eigenständige und eigenverantwortliche Lernen von Schüler*innen und Studierenden zu befördern, sodass der Lehrperson weniger die Wissensvermittlung obliegt, sondern sie eher die Rolle des Lernbegleitenden einnimmt. Außerdem wird darauf abgezielt „die Präsenzzeit für das gemeinsame Üben, Vertiefen und Anwenden des zuvor Gelernten“ (Zickwolf/Kauffeld 2020, S. 46) intensiver zu nutzen.

Allerdings liegen bisher „kaum fächerübergreifende und quantitativ breit aufgestellte Studien“ (ebd., S. 49) zur Wirksamkeit von Flipped-Classroom-Settings vor. Hinzukommt, dass „sich die zur Verfügung stehenden Veröffentlichungen in ihren Ergebnissen widersprechen“ (ebd., S. 49), sodass gegenwärtig keine eindeutigen Aussagen im Hinblick auf die Wirksamkeit des Konzepts postuliert werden können (vgl. ebd., S. 49). „To date, there's no scientific research base to indicate exactly how well flipped classrooms work." (Goodwin/Miller 2013, 
S. 79). Folglich müssen zukünftig verstärkt Anstrengungen unternommen werden, um diese Forschungslücke zu schließen.

Charakteristisch für Flipped-Classroom-Settings ist, dass „die Phasen zum Erwerb von Wissensinhalten und die Anwendung des Wissens im Vergleich zum klassischen Hochschulunterricht umgedreht und ,auf den Kopf gestellt', also ,geflippt““(Volk 2020, S. 212) werden. Die Inhaltserschließung und -vermittlung wird somit in das Selbststudium ausgelagert und durch didaktisch aufbereitete Lehr- und Lernmaterialien der Dozierenden, die auf einer digitalen Lernplattform hinterlegt sind, unterstützt (vgl. Reidsema/Hadgraft/Kavanagh 2017). Dadurch können nicht nur unterschiedliche Niveaus hinsichtlich des erforderlichen Vorwissens ausgeglichen werden, sondern Studierende können das Durcharbeiten der Materialien an das individuelle Lerntempo anpassen (vgl. Volk 2020, S. 212).

In der neu konzipierten Vorlesung zur Einführung in qualitative Forschungsmethoden wurde auf Moodle zurückgegriffen: Dort wurden nicht nur Erklärvideos eingebunden, sondern auch Folien, Übungsaufgaben mit entsprechenden Lösungen hinterlegt. Für die Durchführung der synchronen Sitzung wurde das von der Pädagogischen Hochschule Karlsruhe zur Verfügung gestellte Webkonferenztool Webex genutzt, mit dem Veranstaltungen mit großer Teilnehmendenzahl technisch-störungsfrei durchgeführt werden können. Die asynchrone und synchrone Phase müssen dabei - ganz im Sinne von Flipped-Classroom (vgl. Zickwolf/Kauffeld 2020, S. 46) - aufeinander abgestimmt sein.

Der erste Vorlesungstermin findet als synchrone Veranstaltung im digital vermittelten Raum statt. Neben dem Erörtern prüfungsrelevanter Aspekte wird der erste Termin insbesondere dafür genutzt, den Studierenden die organisatorischen Rahmungen und den Ablauf der Flipped-Classroom-Vorlesung zu kommunizieren und ihre Fragen diesbezüglich zu beantworten. Ihnen wird aufgezeigt, dass sie Eigenverantwortung für das Aneignen der Inhalte in der zeitlich vorgelagerten asynchronen Vorbereitungsphase übernehmen müssen, da diese in der synchronen Veranstaltung nicht wiederholend durchgearbeitet werden. Folglich ist das Aufbereiten dieser eine Voraussetzung, um nachhaltig vom synchronen Teil profitieren zu können, da die Inhalte dort vertieft werden und den Studierenden die Möglichkeit eingeräumt wird, das Gelernte mithilfe von Quizfragen zu überprüfen sowie ihre Fragen zu (noch) unklaren Sachverhalten einzubringen. Die vorzubereitenden Lehr- und Lernmaterialien werden den Studierenden jeweils eine Woche zuvor auf Moodle zur Verfügung gestellt, sodass ausreichend Zeit zur Vorbereitung besteht. Zudem wird den Studierenden mit dem Onlinefragetool frag.jetzt eine Plattform bereitgestellt, auf der sie bereits vorab ihre Fragen festhalten können, die sich während der Vorbereitung ergeben (siehe Abb. 1). Diese können zudem im Hinblick auf ihre Relevanz bewertet werden, sodass der Dozent einen Überblick bekommt, welche Fragen besonders dringlich erscheinen.

Im Anschluss an die organisatorische Einführung erfolgt eine erste inhaltliche Hinführung zur Vorlesungsthematik, in der die Studierenden dazu aufgefordert wurden, Vorteile und Nachteile wissenschaftlich bzw. nicht-wissenschaftlich produzierten Wissens auf der Plattform Tricider, einem webbasierten Tool zum Generieren und Bewerten von Ideen, zu formu- 
lieren. Exemplarisch wurden einige Ideen und Antworten aufgegriffen und mit der Vorlesungsthematik in Bezug gesetzt, um den Studierenden aufzuzeigen, warum sie sich als zukünftige Lehrkräfte mit Forschungsmethoden und bildungswissenschaftlichem Wissen, das durch eben jene Methoden generiert wird, auseinandersetzen müssen.

Wie geht man mit der Offenheit in Leitfadeninterviews um? Durch das Formulieren von Leitfragen wird die Offenheit doch eingeschränkt, oder?

\section{$\boldsymbol{\theta} 813$}

Abbildung 1: Oberfläche frag.jetzt

Nachfolgend wird der Blick auf die Rahmung der synchronen Sitzungen der Flipped-Classroom-Vorlesung im digital vermittelten Raum gerichtet, um den konkreten Vollzug, die Didaktisierung und die zur Anwendung kommenden digitalen Tools näher zu betrachten. Um die Studierenden zu aktivieren, beginnt jede Sitzung mit einem Quiz, das vier Fragen umfasst und die vorbereitete Thematik (anwendungsbezogen) aufgreift. Die Fragen sind dabei so gestaltet, dass sie im Hinblick auf das Schwierigkeitsniveau und die Fragetypen (Single-, Multiple Choice und offene Fragen) an die am Vorlesungsende zu schreibende Klausur angelehnt sind. Entsprechend weist das Quiz nicht nur einen verständnisüberprüfenden, sondern ebenso einen klausurvorbereitenden Charakter auf.

\section{Für eine Analyse ziehen Sie Bilderalben und Transkripte von Expert*inneninterviews heran. Um welche} Form der Triangulation handelt es sich nach Denzin?

\begin{tabular}{|l|l|}
\hline & Wähle die richtige Antwort. \\
\hline 0 & Methoden-Triangulation \\
\hline 0 & Daten-Triangulation \\
\hline 0 & Theorien-Triangulation \\
\hline & Forschenden-Triangulation \\
\hline
\end{tabular}

Abbildung 2: Oberfläche Quiz Academy

Durchgeführt wird das Quiz mit der Plattform Quiz Academy (siehe Abb. 2), die eine Bestenliste visualisiert, sodass das Quiz einen gewissen Spielcharakter aufweist. Im Anschluss an jede 
Frage geht der Dozent auf diese und die Antwortmöglichkeiten kurz ein und erklärt bezugnehmend auf die vorbereiteten Erklärvideos und Folien, warum die Antworten richtig bzw. falsch sind.

Im Anschluss an das Quiz wird den Studierenden Raum gegeben, ihre individuellen Fragen einzubringen. Neben den vorab festgehaltenen Fragen auf der Plattform frag.jetzt haben die Studierenden ebenso die Möglichkeit, spontan oder wenig ausformulierte Fragen im Vorlesungsplenum zu stellen. Obwohl die Fragen, die über die Plattform eingereicht werden, hinsichtlich ihrer Relevanz ausgewählt werden, wird dennoch darauf geachtet, auch den eher unmittelbaren Fragen Raum zu gegeben, sodass das Aufgreifen im Wechsel erfolgt.

Um die Interaktivität mit und unter den Studierenden zu intensivieren, werden diese aktiv in die synchrone Vorlesungssitzung mit einbezogen. Das Beantworten der studentischen Fragen erfolgt nicht ausschließlich durch den Dozenten, sondern dieser spielt eine Vielzahl an Fragen zurück an die Studierenden. Ihnen wird u.a. mittels Breakout-Rooms in Kleingruppen die Möglichkeit eingeräumt, die Fragen oder auch Thesen, die der Dozent zur Diskussion stellt, zu besprechen und sich dazu auszutauschen. Anschließend werden die Ergebnisse ins Vorlesungsplenum getragen. Damit soll nicht nur der Austausch unter den Studierenden, sondern ebenso die Soziabilität, die aufgrund des Digitalen oftmals in den Hintergrund tritt, befördert werden. Ferner wird ebenso darauf abgezielt, dass Studierende das Gelernte in eigenen Worten (zunächst geschützt in den Breakout-Rooms) wiedergeben, sie also entsprechend ihr Wissen in die Vorlesungssitzung mit einbringen und anderen vermitteln, und zugleich überprüfen können, inwiefern sie Sachverhalte richtig verstanden haben. In den Breakout-Rooms werfen die Studierenden zudem oftmals gemeinsam weiterführende Fragen auf, die im Plenum entsprechend vertieft werden. Mit dieser didaktischen Rahmung wird nicht nur verfolgt, eine abwechslungsreiche Lehr-Lern-Umgebung im digital vermittelten Raum zu schaffen, die zur Erhöhung der Aufmerksamkeit beiträgt, sondern ebenso, dass sich Inhalte durch das studentische Erklären nachhaltig verfestigen: sowohl bei jenen, die entsprechende Ausführungen tätigen, als auch bei denen die versuchen, die Erläuterungen nachzuvollziehen.

Basierend auf den gemachten Erfahrungen der digitalen Methodenlehre lässt sich aus Sicht des Dozenten festhalten, dass die Studierenden den Raum in den synchronen Sitzungen rege genutzt haben. Es wurden vielfältige weiterführende Fragen sowie Gesichtspunkte zu denen noch Erklärungsbedarf bestand von den Studierenden eingebracht. Auch die Teilnahme am Quiz zeichnet eine hohe Beteiligung aus. Insgesamt wurde somit an den Lernverläufen und -bedürfnissen der Studierenden angesetzt. Neben der Aufbereitung der Lerninhalte, die zunächst recht zeitaufwendig sind, erfordern insbesondere die synchronen Sitzungen ein hohes Maß an Flexibilität und Spontanität vom Dozenten, da bspw. abgewogen werden muss, inwiefern es produktiv erscheint, dass Fragen zunächst von den Studierenden peer-to-peer in Breakout-Rooms diskutiert werden oder direkt im Vorlesungsplenum vom Dozenten oder den Studierenden beantwortet werden sollten. Entsprechend kann nicht nur eine gewisse Methodenvielfalt als produktiv erachtet werden, sondern ebenso eine gewisse Sensibilität im Hinblick auf die aktuellen (Lern- und Kommunikations-)Bedürfnisse der Studierenden. 
Ohne ausführlich auf die Lehrevaluation der Veranstaltung einzugehen, so wird nachfolgend dennoch exemplarisch die Studierendensicht veranschaulicht. Diese zeigt, dass der Großteil der Studierenden die eingesetzten Online-Tools und den Wechsel dieser innerhalb der synchronen Sitzung als überaus angemessen aufgefasst haben (87\%). Das zielorientierte Einsetzen der digitalen Tools, wie Lernvideos und entsprechende Foliensätze, die aus Sicht der Studierenden rechtzeitig zur Verfügung gestellt wurden (93\%), wird ebenso als überaus positiv bewertet (73\%). Auch die Interaktionen innerhalb des digital vermittelten Raums werden als gelungen bewertet (86\%). Darüber hinaus erachten 68\% der Studierenden das digitale Setting als eine Alternative zur Präsenzvorlesung. Trotz der überwiegend positiven Rückmeldungen der Studierenden müssen jedoch auch potenzielle Schwachstellen, wie bspw. die synchronen Sitzungen weiter gerahmt werden können oder welche weiteren digitalen Tools produktiv in die asynchronen und synchronen Sitzungen implementiert werden können, in den Blick genommen werden, um die Methodenlehre im digital vermittelten Raum weiter zu optimieren.

\subsection{Forschungswerkstatt „Biografische Fallrekonstruktion “ im digital vermittelten Raum}

Die Forschungswerkstatt biografische Fallrekonstruktion ist ein fester Bestandteil des Angebots zu empirischen Forschungsmethoden der Pädagogischen Hochschule Karlsruhe und seit 2018 am hiesigen Institut für Bildungswissenschaftliche Forschungsmethoden verankert. Im Fokus steht die gemeinsame forschungspraktische Analysearbeit am Datenmaterial. Die gemeinsame Arbeitsweise erfolgt in einem interdisziplinären und statusübergreifenden Austausch und richtet sich insbesondere an Promovierende, Habilitierende und Mitarbeitende. Jedoch steht die Forschungswerkstatt auch für interessierte Bachelor- und Masterstudierende offen.

Von den Teilnehmenden wird erwartet, dass sie kontinuierlich und über einen Zeitraum von mindestens zwei Semestern mitwirken, sich auf die entsprechenden Datenmaterialien einlassen, diese vorbereiten und sich aktiv an den Gesprächen bzw. der Analysearbeit der Forschungswerkstatt beteiligen. Mit dem kollaborativen Arbeiten wird darauf abgezielt, die Teilnehmenden aktiv in das Analysieren von biografischem Datenmaterial zu involvieren, sodass neben dem Vermitteln der technischen Analyseinstrumentarien ebenso die Interpretationspraxis am Datenmaterial und der Umgang mit der Vieldeutigkeit qualitativer Daten habitualisiert werden kann. Diese Arbeitsweise verhilft zudem, „zu einer nachvollziehbaren - im Sinne einer intersubjektiv geteilten - Deutung“ (Tiefel/Kondratjuk 2021, S. 211) sowie zu einer „Differenzierung und Verdichtung von analytischen Abstraktionen, kontrastiven Vergleichen und theoretischen Modellen“ (Riemann 2011, S. 413) zu gelangen.

Vorbereitend auf das synchrone Treffen in der Forschungswerkstatt bearbeiten die Teilnehmenden ein anonymisiertes biografisch-narratives Interview, das vor dem Hintergrund einer spezifischen Forschungsfrage analytisch betrachtet wird und den Teilnehmenden eine Woche im Voraus zukommt. Dieses bildet die Grundlage für die gemeinsame Interpretation im digital vermittelten Raum. Für das Durchführen des synchronen Treffens wird ebenso auf das Webkonferenztool Webex zurückgegriffen. Da die Teilnehmenden der Forschungswerkstatt bereits über einen längeren Zeitraum an den gemeinsamen Interpretationssitzungen partizipie- 
ren sind sie bestens mit den kommunikativen Interaktionen und Abläufen innerhalb der relativ fest zusammengesetzten Gruppe vertraut. Entsprechend gestaltete sich der Wechsel in den digital vermittelten Raum relativ reibungslos. Mit Herausforderungen war jedoch die Integration neuer Teilnehmer*innen verbunden, - die weder mit den kommunikativen Abläufen und Interaktionen der Forschungswerkstatt vertraut waren noch einzelne Personen der bestehenden Gruppe kannten. Aufgrund der fehlenden leiblichen Präsenz bedarf diese im digital vermittelten Raum einer stärkeren Rahmung, auf die nachfolgend eingegangen wird.

Die Begrüßung neuer Teilnehmer*innen in der Forschungswerkstatt erfolgt - ähnlich wie in Präsenzsitzungen - zunächst mit einer Vorstellungsrunde in der alle etwas zur eigenen Person, zu ihren Forschungsinteressen und zu ihrem gegenwärtigen Forschungsvorhaben erzählen. Im Anschluss daran kommuniziert die Werkstättenleitung allgemeines zu Forschungswerkstätten und deren Rahmungen und geht auf sich ergebende Fragen der neuen Teilnehmenden ein.

Trotz des kommunikativen Austauschs und der damit verbundenen Nähe im digital vermittelten Raum bedarf es in der Phase der Dateninterpretation, also der gemeinsamen Auseinandersetzung mit dem Datenmaterial, aufgrund der physischen und leiblichen Distanz einer besonderen Aufmerksamkeit für neue Teilnehmende, um so einem Zurückziehen, das im digital vermittelten Raum grundsätzlich einfacher ist, aktiv entgegenzuwirken. Dies erfolgt u.a. durch ein direktes Ansprechen neuer Teilnehmenden durch die Werkstättenleitung: bspw. wie sie über eine zuvor angelegte Interpretation denken und inwiefern sie den Sachverhalt ähnlich oder anders gelagert betrachten. Wichtig dabei ist wiederholt aufzuzeigen, dass es nicht nur eine Lesart und Deutung, sondern unterschiedliche gibt, die es einzubringen gilt und über die sich ausgetauscht wird, wie die nachfolgende Äußerung der Werkstattleitung verdeutlicht: „Danke für diese spannende Sichtweise, die ich bisher noch gar nicht so im Blick hatte. Wir können diese gerne weiterverfolgen“. Auf diese Art und Weise wird nicht nur Partizipation ermöglicht, sondern ebenso dazu beigetragen, eine Atmosphäre vertrauensvoller Verständigung frei von „professionelle[m] Gehabe“ (Allert et al. 2014, S. 295) zu schaffen und die neuen Teilnehmenden in die Gruppe sowie in die kommunikativen Abläufe und Interaktionen im digital vermittelten Raum zu integrieren.

Im digital vermittelten Raum haben sich die neuen Teilnehmenden aktiv an den Diskussionen beteiligt sowie ihre Interpretationen und Positionen produktiv eingebracht. Ferner haben sie nicht nur weiterhin an der Forschungswerkstatt teilgenommen, sie haben sich also nicht zurückgezogen, sondern teilweise auch eigenes Datenmaterial eingebracht.

\section{Fazit}

Die Ausführungen veranschaulichen, dass sich unterschiedliche Veranstaltungsformate qualitativer Methodenlehre im digital vermittelten Raum umsetzen lassen und auch Interaktionen, (verbale) Kommunikationen und die gemeinsame Arbeit am Datenmaterial, die für die Vermittlung qualitativer Forschungsmethoden als zentral angesehen werden, durch spezifisch didaktische Rahmungen realisiert werden können. Es wurden zwar eigene Erfahrungen und Rückmeldungen aus der Lehrevaluation veranschaulicht, allerdings sind dennoch weitere Evaluationen, auch im Sinne eines Längsschnitts notwendig, um differenziertere Aussagen nicht 
nur im Hinblick auf die Wirksamkeit treffen zu können, sondern ebenso inwiefern die digitale Methodenlehre anschlussfähig an die digitale(n) Lebenswelt(en) der Studierenden ist und sie diese sowie ihre einzelnen Elemente als gelungen auffassen. Offen bleibt allerdings, inwiefern deren Intensität und Qualität im Vergleich zu Präsenzveranstaltungen eine andere ist und ob das digitale Setting auch einen Beitrag zum Ausbilden der eingangs erwähnten Schlüsselqualifikationen leistet. Basierend auf bisherigen Befunden zur Wirksamkeit von Flipped-Classroom-Settings (vgl. Zickwolf/Kauffeld 2020, S. 46) kann vermutet werden, dass auch im Rahmen dieser Schlüsselqualifikationen angeregt werden. Dies gilt es zukünftig verstärkt in den Blick zu nehmen. Obwohl die Flipped-Classroom-Vorlesung insbesondere für den Dozenten mit einem erheblichen zeitlichen Vorbereitungsaufwand verbunden ist, so bietet sie für Studierende verstärkt die Möglichkeit, (offene) Fragen im synchronen Treffen einzubringen, die in entsprechender inhaltlichen Tiefe ausgeführt und mit zusätzlichen Praxisbeispielen angereichert werden können. Zudem können sie sich ,unbehelligt' von dem Dozierenden mit den Lehrinhalten und Übungsaufgaben auseinandersetzen, diese vorbereiten, die Materialien im eigenen Lerntempo durcharbeiten und immer wieder die Ausführungen des Dozierenden in den Erklärvideos heranziehen. Entsprechend weist das Format des Flipped-Classroom auch für die Zeit nach der Corona-Pandemie ein erhebliches Potenzial auf, um angemessen auf die Bedürfnisse der Studierenden einzugehen, das es weiter auszuschöpfen gilt. Ähnliches kann für die Forschungswerkstatt festgehalten werden: Der digitale Raum ermöglichte plötzlich die Teilnahme für Personen, die zwar Interesse, jedoch aufgrund der räumlichen Distanz bisher nicht die Möglichkeit hatten, an der Forschungswerkstatt teilzunehmen. So konnte bspw. eine Person, die im skandinavischen Raum ihren Lebensmittelpunkt hat, ihre Teilnahme realisieren. Folglich kann die Umsetzung von Forschungswerkstätten im digital vermittelten Raum ebenso dazu beitragen, dass die Heterogenität der Gruppe zunimmt und somit auch eine größere Bandbreite an unterschiedlichen Perspektiven vorhanden ist, als wenn sie in Präsenz durchgeführt werden. Auch hier gilt es, entsprechende Potenziale unter Berücksichtigung von Limitierungen weiter auszuschöpfen, sodass zukünftig Hybridmodelle angedacht sind, insofern es die technische Ausstattung zulässt.

\section{Literatur}

Abeysekera, Lakmal/Dawson, Phillip (2014): Motivation and cognitive load in the flipped classroom: Definition, rationale and a call for research. In: Higher Education Research \& Development, 34(1), S. 1-14. URL: doi.org/10.1080/07294360.2014.934336.

Allert, Tilman/Dausien, Bettina/Mey, Günter/Reichertz, Jo/Riemann, Gerhard (2014): Forschungswerkstätten - Programme, Potenziale, Probleme, Perspektiven. Eine Diskussion. In: Mey, Günter/Mruck, Katja (Hrsg.): Qualitative Forschung. Analysen und Diskussionen, Wiesbaden: Springer, S. 291-316.

Beutel, Mirja/Floß, Peter/Kull, Carolin/Wigger-Eckei, Aline (2020): Forschendes Lernen in den Praxisphasen des Lehramtsstudiums. Ein Beitrag zur Entwicklung professioneller Überzeugungen. In: Wulf, Carmen/Haberstroh, Susanne/Petersen, Maren (Hrsg.): Forschendes Lernen. Theorie, Empirie, Praxis. Wiesbaden: Springer VS, S. 111-128. 
Breuer, Franz/Schreier, Margrit (2007): Zur Frage des Lehrens und Lernens von qualitativ-sozial-wissenschaftlicher Forschungsmethodik. In: Forum Qualitative Sozialforschung/Forum: Qualitative Social Research, 8 (1), Art. 30. URL: doi.org/10.17169/fqs-8.1.216.

Dausien, Bettina (2007): Reflexivität, Vertrauen, Professionalität. Was Studierende in einer gemeinsamen Praxis qualitativer Forschung lernen können. In: Forum Qualitative Sozialforschung/Forum: Qualitative Social Research, 8 (1). URL: doi.org/10.17169/fqs-8.1.220.

Fabel-Lamla, Melanie/Pietsch, Susanne (2012): Vom Beobachten zum Handeln im Lehrerberuf. Herausforderungen und Prozessstrukturen bei der Bearbeitung pädagogischer Problemsituationen. In: Boer, Heike de/Reh, Sabine (Hrsg.): Beobachtung in der Schule - Beobachten lernen. Wiesbaden: Springer, S. 281-300.

Goodwin, Bryan/Miller, Kirsten (2013): Research says/Evidence on flipped classrooms is still coming in. In: Educational Leadership, 70(6), S. 78-80.

Jütte, Wolfgang/Walber, Markus/Lobe, Claudia (2016): Hochschulbezogene Lehr-/Lern-Forschung als Basis für die Lehrprofessionalisierung. In: Brahm, Taiga/Jenert, Tobias/Euler, Dieter (Hrsg.): Pädagogische Hochschulentwicklung. Wiesbaden: Springer, S. 83-99.

Kanter, Heike/Mey, Günter (2021): Herausforderungen, qualitative Forschungsmethoden zu lehren/lernen. Ansprüche, Spezifika und Lösungswege zum Erwerb von Schlüsselqualifikationen an Hochschulen der angewandten Wissenschaft. In: Kunz, Alexa Maria/Mey, Günter/Raab, Jürgen/Albrecht, Felix (Hrsg.): Qualitativ Forschen als Schlüsselqualifikation. Prämissen - Praktiken - Perspektiven. Weinheim: Beltz Juventa, S. 26-51.

Kerres, Michael/Preußler, Annabell (2013): Zum didaktischen Potenzial der Vorlesung: Auslaufmodell oder Zukunftsformat? In: Reinmann, Gabi/Ebner, Martin/Schön, Sandra (Hrsg.): Hochschuldidaktik im Zeichen von Heterogenität und Vielfalt. Doppelfestschrift für Peter Baumgartner und Rolf Schulmeister. Bad Reichenhall: BIMS, S. 79-98.

Kunz, Alexa Maria/Mey, Günter/Raab, Jürgen/Albrecht, Felix (2020): Qualitativ Forschen als Schlüsselqualifikation. Prämissen - Praktiken - Perspektiven. Weinheim: Beltz Juventa.

Loviscach, Jörn (2020): Inverted Classroom Model: mehr als nur eine Vorbereitung mit Videos. In: Kauffeld, Simone/Othmer, Julius (Hrsg.): Handbuch Innovative Lehre. Wiesbaden: Springer, S. 87-98.

Mey, Günter (2008): Lehre (in) der Qualitativen Forschung - eine Leerstelle? In: Journal für Psychologie 16 (1), URL: journal-fuer-psychologie.de/index.php/jfp/article/view/194/, S. 1-13.

Owston, Ron (2013): Blended learning policy and implementation: Introduction to the special issue. In: Internet and Higher Education, 18, pp. 1-3.

Panenka, Petra/Epp, André/Kunina-Habenicht, Olga (2021): Die Aneignung der Schlüsselqualifikation (Selbst-)Reflexion in der Qualifikation von Tutor*innen für qualitative Forschungsmethoden. In: Kunz, Alexa Maria/Mey, Günter/Raab, Jürgen/Albrecht, Felix (Hrsg.): Qualitativ Forschen als Schlüsselqualifikation. Prämissen - Praktiken - Perspektiven. Weinheim: Beltz Juventa, S. 124-150.

Pfaff, Nicolle/Tervooren, Anja (2020): Qualitatives Methodenlernen im Kontext digitaler Medien. Editorial. In: ZQF - Zeitschrift für Qualitative Forschung, 21 (2), S. 157-161.

Reidsema, Carl/Hadgraft, Roger/Kavanagh, Lydia (2017): Introduction to the flipped classroom. In: Reidsema, Carl/Kavanagh, Lydia/Hadgraft, Roger/Smith, Neville (Eds.): The flipped classroom practice and practices in higher education. Singapore: Springer, pp. 3-14.

Riemann, Gerhard (2011): Grounded theorizing als Gespräch. Anmerkungen zu Anselm Strauss, der frühen Chicagoer Soziologie und der Arbeit in Forschungswerkstätten. In: Mey, Günter/Mruck, Katja (Hrsg.): Grounded Theory Reader. Wiesbaden: VS Verlag für Sozialwissenschaften/Springer Fachmedien Wiesbaden, S. 405-426. 
Ruppel, Paul Sebastian (2020): Die Forschungswerkstatt als kooperatives Lehr-Lern-Arrangement: Potenziale und Herausforderungen einer hybriden Form der On- und Offline-Zusammenarbeit. In: ZQF - Zeitschrift für Qualitative Forschung, 21 (2), S. 217-232.

Schäfer, Anna Maria (2012): Das Inverted Classroom Model. In: Handke, Jürgen/Sperl, Alexander (Hrsg.): Das Inverted Classroom Model. Begleitband zur ersten deutschen ICM Konferenz. München: Oldenbourg, S. 3-11.

Schrader, Tina-Berith/Brenneke, Bettina/Pfaff, Nicolle/Tervooren, Anja (2020): „Das ist jetzt eine gute Situation und das kannst du gut beobachten...". Rekonstruktionen studentischer Erfahrungen des qualitativen Methodenlernens als Ausgangspunkte der Entwicklung von forschungsmethodischen Online-Kursen. In: ZQF - Zeitschrift für Qualitative Forschung, 21 (2), S. 199-215.

Schreier, Margrit/Breuer, Franz (2020): Lehren und Lernen qualitativer Forschungsmethoden in der Psychologie. In: Mey, Günter/Mruck, Katja (Hrsg.): Handbuch Qualitative Forschung in der Psychologie, Wiesbaden: Springer, S. 265-289. URL: doi.org/10.1007/978-3-658-18387-5_32.

Sein-Echaluce, Maria Luisa/Fidalgo-Blanco, Ángel/García-Peñalvo, Francisco (2019): Innovative trends in flipped teaching and adaptive learning. Hershey: IGI Global, URL: doi.org/10.4018/9781-5225-8142-0.

Spies, Anke/Knapp, Katja (2020): Forschendes Lernen in der ersten Phase der Lehrkräftebildung. Retrospektive Deutungen zur Nachhaltigkeit einer Lernerfahrung. In: Wulf, Carmen/Haberstroh, Susanne/Petersen, Maren (Hrsg.): Forschendes Lernen. Theorie, Empirie, Praxis. Wiesbaden: Springer, S. 145-156.

Tiefel, Sandra/Kondratjuk, Maria (2021): Vagheit und Tentativität als bildungsrelevante Schlüsselkompetenzen. Reflexionen über die Praxis der Forschungswerkstatt zur qualitativen Forschung auf Basis von Anselm Strauss' Theorie sozialer Welten und Arbeitsbogen-Konzept. In: Kunz, Alexa Maria/Mey, Günter/Raab, Jürgen/Albrecht, Felix (Hrsg.): Qualitativ Forschen als Schlüsselqualifikation. Prämissen - Praktiken - Perspektiven. Weinheim: Beltz Juventa, S. 202-219.

Volk, Benno (2020): Vorlesungen vor dem Hintergrund aktueller Flipped Classroom-Ansätze. In: Egger, Rudolf/Eugster, Balthasar (Hrsg.): Lob der Vorlesung. Vorschläge zur Verständigung über Form, Funktion und Ziele universitärer Lehre. Wiesbaden: Springer, S. 205-226.

Zwickwolf, Katharina/Kauffeld, Simone (2020): Inverted Classroom. In: Kauffeld, Simone/Othmer, Julius (Hrsg.): Handbuch Innovative Lehre. Wiesbaden: Springer, S. 45-52. 


\section{Informationen zum Autor}

Dr. André Epp ist wissenschaftlicher Mitarbeiter am Institut für Bildungswissenschaftliche Forschungsmethoden der Pädagogischen Hochschule Karlsruhe. Er promovierte an der Universität Hildesheim zu den subjektiven Theorien von Lehrkräften über ungünstige Faktoren in der Bildungsbiografie von Schülerinnen und Schülern und den sozialen Konstruktionen der Lehrkräfte. Darüber hinaus hat er den Promotionsstudiengang Qualitative Bildungs- und Sozialforschung an der Universität Magdeburg erfolgreich abgeschlossen. Forschungsschwerpunkte: Professions- und biografische Lehrer*innen(bildungs)forschung, qualitative Sozialforschung und ihre theoretischen Grundlagen, Übergänge im Bildungssystem, Kinder- und Jugendarbeit, non-formales und informelles Lernen, NichtTraditionelle Studierende, Subjektive Theorien und Alltagstheorien.

andre.epp@ph-karlsruhe.de

\section{Zitationshinweis:}

Epp, André (2021): Vermittlung qualitativer Forschungsmethoden im digitalen Raum - (Didaktische) Einblicke in eine bisher wenig thematisierte Praxis der Hochschullehre. In: Online-Magazin Ludwigsburger Beiträge zur Medienpädagogik, Ausgabe 21/2021. URL: medienpaed-ludwigsburg.de/ 